\title{
Induced Universal Properties and Deconfinement
}

\author{
Ágnes Mócsy * Francesco SAnnino \\ The Niels Bohr Institute \& NORDITA, Blegdamsvej 17, DK-2100 Copenhagen Ø, Denmark
}

(Dated: May 2003)

\begin{abstract}
We propose a general strategy to determine universal properties induced by a nearby phase transition on a non-order parameter field. We use a general renormalizable Lagrangian, which contains the order parameter and a non-order parameter field, and respects all the symmetries present. We investigate the case in which the order parameter field depends only on space coordinates, and the case in which this field is also time dependent. We find that the spatial correlator of the non-order parameter field, in both cases, is infrared dominated and can be used to determine properties of the phase transition. We predict a universal behavior for the screening mass of a generic singlet field, and show how to extract relevant information from such a quantity. We also demonstrate that the pole mass of the non-order parameter field is not infrared sensitive. Our results can be applied to any continuous phase transition. As an example, we consider the deconfining transition in pure Yang-Mills theory, and show that our findings are supported by lattice data. Our analysis suggests that monitoring the spatial correlators of different hadron species, more specifically the derivatives of these, provides an efficient and sufficient way to experimentally uncover the deconfining phase transition and its features.
\end{abstract}

\section{INTRODUCTION}

The relevant properties of phase transitions are best investigated using order parameters. However, sometimes it is profitable to determine new universal features associated with non-order parameter fields [1, 2]. Besides, in nature most fields are non-order parameter ones. To derive the general properties of a generic singlet field we couple this to the order parameter [2]. In this way we can directly study the transfer of information, envisioned in [1], of the phase transition properties from the order parameter to the singlet field.

We analyze two cases: time dependent order parameter fields and time independent ones. The non-order parameter field, always time dependent, is a scalar under Lorentz transformations. Also, it is a singlet under any symmetry group, in particular under the symmetry group whose breaking is monitored by the order parameter. We further assume the non-order parameter field to have a large mass with respect to all the other scales in play, and hence, an associated small correlation length near the phase transition. Although time independent order parameter fields carry the physical information about the phase transition, they do not propagate and cannot be canonically quantized. An example is the Polyakov loop, which is considered to be the order parameter of the pure Yang-Mills theory, and by construction is a function only of space. A generic singlet field in the Yang-Mills theory is the glueball, which is also a physical state of the theory.

Time dependent order parameter fields are generally associated with physical states. These can be either composite, such as the chiral condensate, the order parameter related to chiral symmetry in QCD, or elementary, such as the Higgs field might be for the electroweak phase transition. Using general field theoretical arguments we demonstrate that the screening mass associated with the spatial two-point function of the singlet field is heavily affected by the nearby phase transition for both time dependent and time independent order parameters. The important fact is that we can predict the general behavior of the static two-point function of the singlet field, and associate it uniquely with the specific character of the phase transition. More specifically, the screening mass at one loop has a drop near the phase transition. This is due to the three dimensional nature of the screening mass, which makes it particularly sensitive to infrared physics, and it explains the transfer of information between the order parameter and the singlet field. When going beyond the one loop approximation the drop of the screening mass of the scalar singlet field, both for time independent and time dependent order parameters, is finite at the phase transition. Furthermore, the drop itself is controlled by the ratio of the square of the relevant coupling of the singlet field to the order parameter and the coupling governing the self-interaction of the order parameter. The result for the three dimensional order parameter has already been presented in 2]. Here we provide details of the basic computations used in [2], especially for the higher order corrections, as well as a proof of the result for the screening mass in the time dependent case. For the

\footnotetext{
*Electronic address: mocsy@alf.nbi.dk

${ }^{\dagger}$ Electronic address: francesco.sannino@nbi.dk

${ }^{\ddagger}$ Electronic address: tuominen@nordita.dk
} 
latter we also present the effects of the nearby phase transition on the pole mass of the singlet field. We demonstrate, somewhat surprisingly, that although we still observe a small drop, this physical quantity is not infrared dominated. So in general, only the spatial correlation lengths feel the presence of a nearby phase transition.

The paper is organized as follows: In section III we introduce the theory which is investigated in detail in section III for the case of an order parameter field that depends only on the space coordinates, and in section IV for an order parameter field that depends both on time and space. The information conveyed in section III complements and enlarges the one presented in ref. [2]. In section $\nabla$ we conclude.

Our main conclusion in both cases, space and space-time dependent order parameter fields, is that the information about the phase transition, encoded in the behavior of the order parameter field is transferred to, and obtainable from the singlet field(s) present in the theory. The present analysis suggests that monitoring the spatial correlators even of heavy hadrons provides an efficient and sufficient experimental way to uncover the existence and features of the chiral/deconfining phase transition. So if our results are phenomenologically applicable in relativistic heavy ion collisions at RHIC, they would lead to a clear signal for the existence of a deconfined phase at the early instant of such a collision.

\section{GENERAL SET UP}

We consider a temperature driven phase transition and work in a regime close to the phase transition. In order for our results to be as universal as possible, we use a renormalizable Lagrangian containing a field neutral under the global symmetries, and the order parameter field, charged under the symmetries, as well as their interactions. The protagonists of our theory are two real fields, $h$ and $\chi$. The field $h$ is a scalar singlet, while $\chi$ transforms according to $\chi \rightarrow z \chi$ with $z \in Z_{N}$.

While the generalization to $Z_{N}$ is straightforward, we consider explicitly the case of $Z_{2}$, which is suitable for understanding the deconfining phase transition of two color Yang-Mills. This has been heavily studied via lattice simulations [3, 4]. The most general renormalizable potential is:

$$
V(h, \chi)=\frac{m^{2}}{2} h^{2}+\frac{m_{0 \chi}^{2}}{2} \chi^{2}+\frac{\lambda}{4 !} \chi^{4}+g_{0} h+\frac{g_{1}}{2} h \chi^{2}+\frac{g_{2}}{4} h^{2} \chi^{2}+\frac{g_{3}}{3 !} h^{3}+\frac{g_{4}}{4 !} h^{4} .
$$

The coefficients are all real and stability requires $\lambda \geq 0$ and $g_{4} \geq 0$. We further assume $g_{1}>0$ and $g_{0}<0$. At this level we did not yet commit on the space-time dependence of the fields, although we have normalized them as if they were living in $3+1$ dimensions. This renormalizable potential can be considered, for example, as a truncation of the one presented in 1], and can be used to determine some of the space-time independent properties of the vacuum. In order to go beyond constant field approximation, we must specify the kinetic terms. Since the singlet field $h$ is physical, it has the ordinary standard four dimensional kinetic term, i.e. $\partial_{\mu} h \partial^{\mu} h$. For the $\chi$ field we first consider the case in which $\chi$ is time independent. In this case the associated kinetic term reads as $\nabla \chi \nabla \chi$. We will then consider the case in which $\chi$ experiences also time dimension, and thus the kinetic term is $\partial_{\mu} \chi \partial^{\mu} \chi$. The time and space independent order parameter field, and its interactions with a singlet field were introduced in [1], in order to understand the transfer of information from Polyakov loops to glueballs in pure Yang-Mills theories. The first studies of a renormalizable version of the previous idea, in which the order parameter is a function only of the space dimensions, were performed in [2]. Here we review and extend the analysis of [2], and show the fundamental differences and similarities with respect to a time dependent order parameter field. Before proceeding we state the assumptions under which we conduct our analysis for the time (in)dependent order parameter: i) The $\chi$ field is light close to the transition, hence it dominates the dynamics. ii) The $h$ field is heavy, and thus we can neglect its quantum and Boltzman suppressed thermal loop corrections. The extremum of the linearized potential (in the $h$ field) is for

$$
\langle\chi\rangle^{2}=-6 \frac{m_{\chi}^{2}}{\lambda} \quad \text { with } \quad m_{\chi}^{2} \simeq m_{0 \chi}^{2}+g_{1}\langle h\rangle, \quad \text { and } \quad\langle h\rangle \simeq-\frac{g_{0}}{m^{2}}-\frac{g_{1}}{2 m^{2}}\langle\chi\rangle^{2}
$$

Here $m_{\chi}$ vanishes at the phase transition. Having $g_{1}>0$ and $g_{0}<0$, together with $3 g_{1}^{2}<\lambda m^{2}$, assures the positivity of these expectation values, and with this choice also the extremum of the potential is a minimum. A more complete treatment would require to go beyond the linearized approximation. The temperature dependence of $\langle\chi\rangle$ and $\langle h\rangle$ is qualitatively sketched in figure 1 Note, that in (1) the term linear in $h$ is removed, at tree level, by shifting the field as follows:

$$
h \rightarrow\langle h\rangle+h .
$$




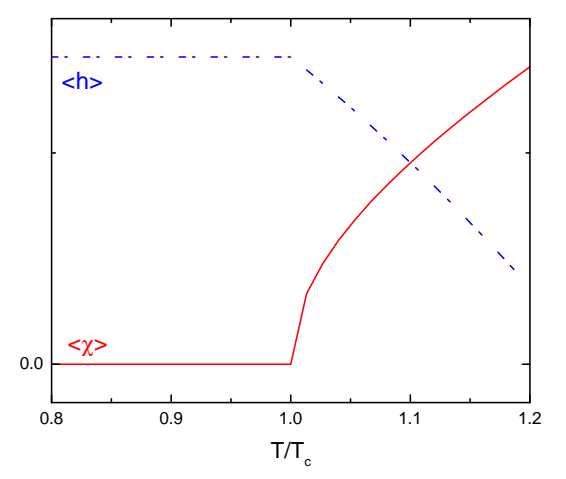

FIG. 1: Schematic behavior of the expectation values of the order parameter $\langle\chi\rangle$ (solid-line) and the non-order parameter $\langle h\rangle$ (dashed-line) fields close to the phase transition as a function of the temperature. Note that $\langle h\rangle$ is never zero and the constancy in the unbroken phase is due to the tree level approximation.

Such term, however, is regenerated at higher orders, correcting the $h$ expectation value. The effect of the shift leads to modified coefficients in the Lagrangian. For example:

$$
g_{0} \rightarrow 0, \quad g_{1} \rightarrow g_{1}+g_{2}\langle h\rangle, \quad m_{0 \chi}^{2} \rightarrow m_{0 \chi}^{2}+g_{1}\langle h\rangle+\frac{g_{2}}{2}\langle h\rangle^{2} .
$$

In the following we consider the fluctuations of $h$ around its vacuum expectation value.

\section{TIME-INDEPENDENT ORDER PARAMETER FIELD : PROBING STATIC PROPERTIES}

The first difference between a purely spatial order parameter and a time dependent one is that in the first case no Bose-Einstein distribution emerges for $\chi$ in thermal equilibrium. More specifically, we are allowed by construction to consider only spatial fluctuations. Besides, since we assumed the field $h$ to be heavy compared to the relevant scales at the phase transition, including the temperature, the $h$ induced thermal corrections can be safely neglected. We postulate, as customary, that $m_{\chi}$ is a function of temperature and vanishing at the transition point. Interestingly, since the order parameter field is purely three dimensional, we can freely choose from which direction the symmetry is restored. Higher order temperature corrections cannot reverse the direction of symmetry restoration. As we discuss in detail in the next section, this is not the case if the order parameter field is time dependent. Having in mind the Yang-Mills deconfining phase transition, we assume that the $Z_{2}$ symmetry is restored at low temperatures. Our analysis is not affected when reversing the direction of the transition.

We have chosen $\chi=\chi(\mathbf{x})$, and decomposed the four dimensional field $h$ into its Matsubara modes. After integrating over time the action reduces to an effective three dimensional one. In the three dimensional case the kinetic term for $h$ and its self-interaction terms receive contributions from all Matsubara modes. However, only the zero mode contributes to the $h \chi^{2}$-interaction, which is the one driving the dynamics of the $h$ field close to the phase transition [2], as the $\chi$ field becomes light with respect to $h$. Hence, we confine our discussion here to the theory which features the fields $\chi$ and $h_{0}$, and study the spatial fluctuations. For simplicity, in this section we denote $h_{0}$ by $h$ which is taken to be directly the fluctuation field around its tree level vacuum expectation value. The three dimensional Lagrangian reads:

$$
-\mathcal{L}_{3}=\frac{1}{2} \nabla h \nabla h+\frac{1}{2} \nabla \chi \nabla \chi+\frac{1}{2} m^{2} h^{2}+\frac{1}{2} m_{\chi}^{2} \chi^{2}+T \frac{\lambda}{4 !}\left(\chi^{2}\right)^{2}+\sqrt{T} \frac{g_{1}}{2} h \chi^{2}+T \frac{g_{2}}{4} h^{2} \chi^{2}+\sqrt{T} \frac{g_{3}}{3 !} h^{3}+T \frac{g_{4}}{4 !} h^{4},
$$

where the coupling constants have the same mass dimension of the corresponding four dimensional theory. All of these are real with $\lambda \geq 0$ and $g_{4} \geq 0$.

Using the renormalizable theory defined by the above Lagrangian, we now compute all one-loop corrections. We find the following contributions to the $h$ one point function:

$$
\bigcirc+\longrightarrow=\frac{\sqrt{T}}{8 \pi}\left(g_{1} m_{\chi}+g_{3} m\right)
$$


which leads to temperature dependent corrections to the expectation value of $\langle h\rangle$. We turn our attention to the $h$ two point function. The full expression at one-loop level is given by the following set of diagrams:

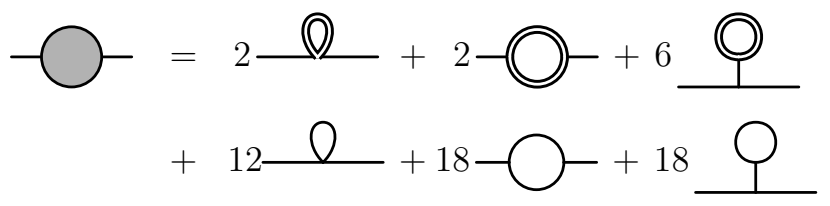

Double lines indicate $\chi$ fields, while single lines stand for the $h$ field. The number in front of each diagram is the associated combinatorial factor. We regularize the ultraviolet divergent contribution by subtracting the divergent terms. The second diagram is infrared divergent for $T \rightarrow T_{c}$ and gives the dominant one loop contribution to the screening mass of $h$. In the limit of zero external momentum:

$$
\bigcirc=T\left(\frac{g_{1}}{2}\right)^{2} \int \frac{d^{3} k}{(2 \pi)^{3}} \frac{1}{\left(k^{2}+m_{\chi}^{2}\right)^{2}}=T \frac{g_{1}^{2}}{32 \pi m_{\chi}} \text {. }
$$

It is worth mentioning that $\langle h(x) h(0)\rangle$ is associated to the correlator $\left\langle\chi^{2}(x) \chi^{2}(0)\right\rangle$ when the couplings $g_{2}, g_{3}$ and $g_{4}$ are neglected since the term $g_{1} h \chi^{2}$ is a source for $\chi^{2}$ when the $h$ kinetic term is omitted.

After having taken proper care of the ultraviolet divergent terms the finite contributions from the diagrams in equation (7) are

$$
m^{2}(T)=m^{2}-T \frac{g_{1}^{2}}{16 \pi m_{\chi}}-\frac{T}{8 \pi} m_{\chi}\left(g_{2}-\frac{g_{1} g_{3}}{m^{2}}\right)-\frac{T}{16 \pi} m\left(2 g_{4}-\frac{g_{3}^{2}}{m^{2}}\right)
$$

which clearly shows that the terms in parenthesis (third and fourth terms) are subleading with respect to the second one near the transition point. Equation (9) illustrates how the nearby phase transition is directly felt by the non-order parameter field, and furthermore, gives the general prediction that the screening mass of the singlet field must decrease close to the phase transition. If we stop the analysis here, at one loop level, we predict the following critical behavior for $\Delta m^{2}=m^{2}(T)-m^{2}$, where $m$ is the mass at a temperature close to the critical point:

$$
\begin{array}{ll}
\Delta m^{2}(T)=-\frac{g_{1}^{2} T}{16 \pi m_{\chi}} \sim t^{-\frac{\nu}{2}}, \quad T<T_{\mathrm{c}} \\
\Delta m^{2}(T)=-\frac{g_{1}^{2} T}{16 \pi M_{\chi}} \sim t^{-\frac{\nu}{2}}, \quad T>T_{\mathrm{c}}
\end{array}
$$

where $M_{\chi}=\sqrt{2}\left|m_{\chi}\right| \propto\left|T-T_{c}\right|^{\nu / 2}$. This one loop result breaks down at the transition point, due to the infrared singularity. However, since $h$ is not the order parameter field, its correlation length (i.e. $1 / m)$ is not expected to diverge at the phase transition. In the following we investigate the behavior of the $h$ screening mass near the phase transition by going beyond the one-loop approximation.

\section{A. Healing the IR behavior}

When analyzing contributions beyond one loop order to the $h$ two-point function, the number of diagrams, and distinct topologies one needs to consider proliferates. We select a subclass of diagrams, that heal the infrared divergences, while capturing the essential physical properties of the problem at hand. Besides, this subclass of diagrams we consider is known to be exact in the large $N$ limit of a theory with $O(N)$ symmetry [5] , and the summation can be performed exactly. We note that a large $N$ approximation might not be the best choice for investigating the details of the phase transition, however we show that it well reproduces the behavior of the screening mass of hadronic degrees of freedom near the deconfinement phase transition as given by lattice simulations. A finite result for the two-point function, both in the broken and unbroken phases will emerge, while the expression for the mass of $h$ turns out to be continuous across $T_{c}$.

In the unbroken phase (i.e. $T<T_{c}$ ) it is known, that for a generic $O(N)$ theory in the large $N$ limit the following chain of bubble diagrams represents the leading contribution,

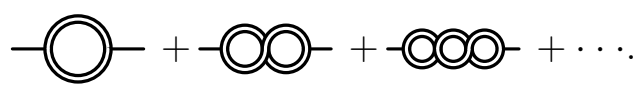


These diagrams constitute a geometrical series. For the $O(N)$ theory this resummation procedure is exact in the large $N$ limit [5]. We choose the same set of diagrams in the unbroken phase. Denoting the dimensionless single loop integral by $\mathcal{I}$, the expression for a single diagram in the chain made of $p$ loops, and $p-1$ vertices of $\chi^{4}$-type is:

$$
\frac{g_{1}^{2}}{2}\left(-\frac{\lambda}{2}\right)^{p-1} \mathcal{I}^{p} .
$$

The exact sum of all the diagrams in the chain is simply:

$$
g_{1}^{2} \mathcal{I} \frac{\frac{1}{2}}{1+\frac{\lambda \mathcal{I}}{2}}, \quad \text { with } \quad \mathcal{I}=\frac{T}{8 \pi m_{\chi}}
$$

Using eq. (13) the following expression for $m$ follows:

$$
m^{2}(T)=m^{2}-T \frac{g_{1}^{2}}{16 \pi m_{\chi}+\lambda T},
$$

which is finite at $T_{c}$, where it yields:

$$
m^{2}\left(T_{c}\right)=m^{2}-\frac{g_{1}^{2}}{\lambda} .
$$

This is the main result of [2], predicting that close to the phase transition the singlet state must have a decreasing mass parameter, associated with spatial correlations. More specifically, the drop at the phase transition point is given by the ratio of the square of the coupling constant governing the interaction of the singlet state with the order parameter (i.e. $g_{1}$ ) to the order parameter field self-interaction coupling constant $\lambda$. In this way, via the drop of the singlet field at the phase transition, one can derive a great deal of information about the phase transition, and about the order parameter itself.

The analysis is much more complicated in the broken phase. Indeed, when $T>T_{c} \chi$ develops an average value $\langle\chi\rangle=v$, which induces one also for $h$ as shown in eq.(2). This is in agreement with the results found in [1]. So on general grounds, the fields $\chi$ and $h$ mix in this phase. Defining the mass eigenstate fields as $H$ and $\Xi$, these are related to $h$ and $\chi$ via:

$$
\left(\begin{array}{l}
h \\
\chi
\end{array}\right)=\left(\begin{array}{cc}
\cos \theta & -\sin \theta \\
\sin \theta & \cos \theta
\end{array}\right)\left(\begin{array}{l}
H \\
\Xi
\end{array}\right) .
$$

The mixing angle $\theta$ is proportional to $g_{1} v / \mathrm{m}^{2}$, and therefore, the mixing can be neglected within the present approximations, simplifying the analysis considerably. Like for the symmetric phase, we consider only the effects due to the $\chi$ loops for the $h$ propagator. Due to symmetry breaking we now need to consider also the trilinear $\chi$ coupling

$$
-\frac{\lambda}{3 !} v \chi^{3},
$$

which is expected to affect the analysis. At one loop level the only diagram to compute is again the one in eq. (8), with $m_{\chi}$ replaced by $M_{\chi}$. Hence, on general grounds, we predict a drop in the mass of $h$ also on the right hand side of $T_{c}$. In this phase the infrared divergence is still present at one loop level. Curing such divergence is now more involved due to symmetry breaking. In the case of the large $N$ limit of $O(N)$ symmetry, one can show, that diagrams with trilinear vertices are again suppressed relative to the simple bubble diagrams.

Here we go beyond the large $N$ limit by computing a new set of diagrams, which can be evaluated exactly, and thus capture relevant corrections due to symmetry breaking, neglected in the large $N$ limit. The new chain of diagrams we compute has terms of the form:

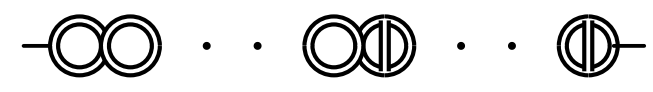

This class of diagrams has knowledge about the onset of the symmetry breaking via the presence of the trilinear vertices, and, in that respect, is the simplest extension of the chain of simple bubble diagrams. Another amusing property of (18) is, that the sum can be performed exactly, as we now demonstrate.

Denoting the number of simple bubbles by $p$, and the number of bubbles with trilinear vertices by $q$, the total number of loops is $L=p+2 q$, and the number of $\chi^{4}$ vertices is $p+q-1$, while that of the trilinear vertices is $2 q$. The loop integral of the single bubble is $\mathcal{I}$. The loop integral of the bubble with two trilinear vertices yields $\mathcal{I}^{2} / 9$. 
The generic single multi-loop diagram of type (18), with $p$ ordinary bubbles and $q$ bubbles with two trilinear vertices, yields the following contribution:

$$
\frac{g_{1}^{2}}{4} 2\left(-\frac{\lambda}{4 !}\right)^{p+q-1}\left(\frac{\lambda v}{3 !}\right)^{2 q}\left(\frac{3}{\lambda v^{2}}\right)^{q} \frac{\mathcal{I}^{p+2 q}}{3^{2 q}}(3 !)^{2 q}\left(\frac{4 !}{2}\right)^{p+q-1}\left(\begin{array}{c}
p+q \\
q
\end{array}\right)=\frac{g_{1}^{2}}{2}\left(-\frac{\lambda}{2}\right)^{p+q-1}\left(\frac{\lambda}{3}\right)^{q} \mathcal{I}^{p+2 q}\left(\begin{array}{c}
p+q \\
q
\end{array}\right) .
$$

On the left hand side of the equation the binomial factor corresponds to the number of different ways to distribute $q$ rungs into $p+q$ loops, and the preceding factorials are the associated combinatorial factors, due to the permutation of the vertices.

The infinite sums over $q$ and $p$ are evaluated by replacing the summation over $p$ with the summation over the total number of loops $L$. For fixed $L, q$ can take values $q=0,1, \ldots,\lfloor L / 2\rfloor$, where $\lfloor x\rfloor$ is the largest positive integer smaller than or equal to $x$. Then the sum becomes

$$
\frac{g_{1}^{2}}{2} \frac{(-2)}{\lambda} \sum_{L=1}^{\infty}\left(-\frac{\lambda \mathcal{I}}{2}\right)^{L} \sum_{q=0}^{q_{\max }}\left(-\frac{2}{3}\right)^{q}\left(\begin{array}{c}
p+q \\
q
\end{array}\right)=\frac{g_{1}^{2}}{2}\left(-\frac{2}{\lambda}\right) \sum_{L=1}^{\infty}\left(-\frac{\lambda \mathcal{I}}{2} \sqrt{\frac{2}{3}}\right)^{L} U_{L}\left(\frac{1}{2} \sqrt{\frac{3}{2}}\right),
$$

where the function $U_{L}(x)$ appearing in the intermediate step is the Chebychev polynomial of the second kind. Remarkably, the final sum can be performed exactly, yielding for $T>T_{c}$ again an infrared finite result:

$$
\begin{aligned}
m^{2}(T) & =m^{2}-\frac{g_{1}^{2} \mathcal{I}}{2} \frac{1+\frac{\lambda}{3} \mathcal{I}}{1+\frac{\lambda}{2} \mathcal{I}+\frac{\lambda^{2}}{6} \mathcal{I}^{2}}, \\
\mathcal{I} & =\frac{T}{8 \pi M_{\chi}}
\end{aligned}
$$

We see that $m\left(T_{c}\right)$ from the broken side of the transition equals exactly the one from the unbroken side of the transition, even when departing from the large $N$ limit. The mass squared of $h$ is a continuous function through the phase transition, and the associated correlation length remains finite. This result does not hold order by order in the loop expansion, but only when the infinite sum of the diagrams is performed. Although admittedly, there is ambiguity in selecting the class of diagrams to take into account, guided by the large $N$ limit in the symmetric phase, we considered only the bubble chain of diagrams. However, in the broken phase we chose to resum a new and richer class of diagrams. The new class contains not only the relevant large $N$ contribution, but also carries information related to the spontaneously broken symmetry. This class, although more involved, can be summed exactly.

In order to disentangle relevant properties of the phase transition, we construct slope parameters for the singlet field:

$$
\mathcal{D}^{ \pm} \equiv \lim _{T \rightarrow T_{\mathrm{c}}^{ \pm}} \frac{1}{\Delta m^{2}(T)} \frac{d m^{2}(T)}{d T},
$$

with $\Delta m^{2}\left(T_{c}\right)=g_{1}^{2} / \lambda$. The functional form of $\mathcal{D}^{+}$and $\mathcal{D}^{-}$is the same, provided that the same class of diagrams is resummed on both sides of the transition. In this case we have:

$$
\mathcal{D}^{-}=\frac{16 \pi}{\lambda T_{c}} \lim _{T \rightarrow T_{c}^{-}} \frac{d m_{\chi}}{d T}
$$

in the symmetric phase, and to obtain $\mathcal{D}^{+}$in the broken phase is sufficient to replace $m_{\chi}$ with $M_{\chi}=\sqrt{2}\left|m_{\chi}\right|$. While the mass of $h$ remains finite at $T_{c}$, its slope encodes the critical behavior of the theory. For example, if $m_{\chi}^{2}$ vanishes as $\left(T_{c}-T\right)^{\nu}$ close to the phase transition (with the correlation length $\xi \propto\left|T-T_{c}\right|^{-\nu / 2}$ ), then $\mathcal{D}^{ \pm}$scales with exponent $(\nu / 2-1)$. A difference in the functional form of the slopes may emerge, when on the two sides of the transition different class of diagrams are resummed. Using the wider class of diagrams in the broken phase considered above while retaining just the simple bubble sum in the unbroken we determine:

$$
\mathcal{D}^{+} \simeq-3 \frac{16 \pi}{\lambda T_{c}}\left|m_{\chi}\right| \mathcal{D}^{-} .
$$

This is due to the onset of spontaneous symmetry breaking, i.e. in the broken phase the class of resummed diagrams contains trilinear type of interactions. We identify thus a less singular behavior with respect to the simple sum of bubbles. More specifically, the scaling exponent for $\mathcal{D}^{+}$is now $(\nu-1)$. While the explicit relations between the 

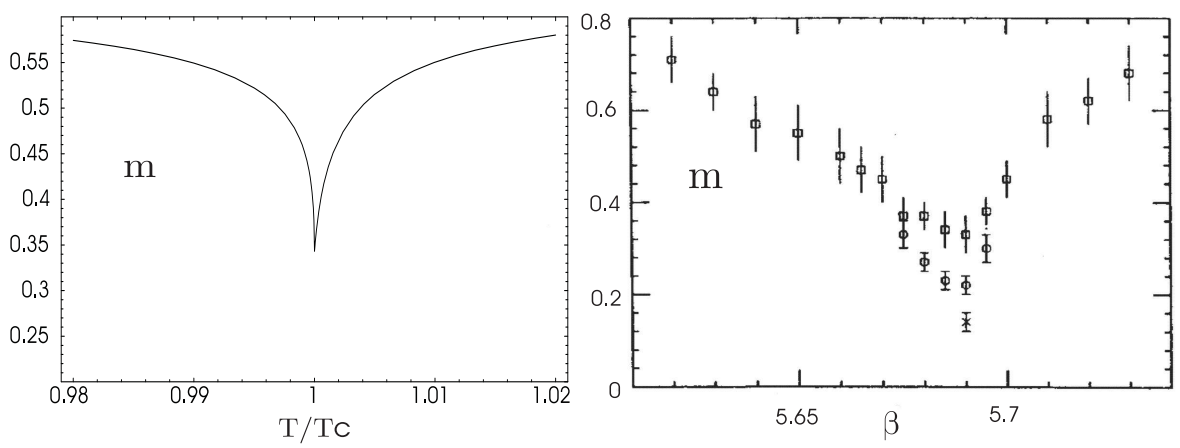

FIG. 2: Left panel: behavior of the mass of the singlet field close to the phase transition as function of the temperature. Right panel: Lattice data; figure from [6].

scaling exponents and the slopes are only valid within the given summation scheme, these quantities are, nevertheless, a measure of the critical behavior near the phase transition. Only experimental results will be able to select which class best describes the data.

In figure 2 we schematically represent the behavior of the $h$ screening mass as a function of the temperature, in units of the critical temperature, for $m_{\chi}^{2} \propto\left(T_{c}-T\right)$. The left panel of fig. [2 illustrates the rapid decrease of the singlet field screening mass in the critical region. The right panel of fig. 20 is the lattice data of [6] obtained for the three color Yang-Mills theory. The mass in [6] is the glueball screening mass. The resemblance between our results and the lattice results is intriguing, but more lattice data is needed in order to distinguish between the above possibilities, and quantitatively determine the size of the drop.

It is common practice to isolate the order parameter field, and more generally, the light degrees of freedom, since they are expected to be the relevant degrees at low energies. While this is certainly correct, we have explicitly shown how the essential features of the phase transition are encoded in the non-order parameter fields of the theory as well. Our analysis is useful whenever the order parameter field is neither a physical quantity, nor is phenomenologically accessible (e.g. the Polyakov Loop).

\section{TIME DEPENDENT ORDER PARAMETER FIELD}

In this section we investigate the effects of the four dimensional $\chi=\chi(\mathbf{x}, t)$ order parameter field on the properties of a singlet $h=h(\mathbf{x}, t)$ field. Some of these effects turn out to be significantly different than for the time independent case. The order parameter field is now a physical field, and as such, it can propagate in time, and can be canonically quantized. The four-dimensional Lagrangian of the renormalizable theory we use to define our Feynman rules is:

$$
\mathcal{L}_{4}=\frac{1}{2} \partial_{\mu} h \partial^{\mu} h+\frac{1}{2} \partial_{\mu} \chi \partial^{\mu} \chi-\frac{m^{2}}{2} h^{2}-\frac{m_{\chi}^{2}}{2} \chi^{2}-\frac{\lambda}{4 !} \chi^{4}-\frac{g_{1}}{2} h \chi^{2}-\frac{g_{2}}{4} h^{2} \chi^{2}-\frac{g_{3}}{3 !} h^{3}-\frac{g_{4}}{4 !} h^{4} .
$$

All coupling constants are real, and $\lambda \geq 0, g_{4} \geq 0$, and $g_{2}$ are dimensionless, while $g_{1}$ and $g_{3}$ have mass dimension one. The largest scale is the mass $m$ of the $h$ field, which, by our assumption, is again much larger than $m_{\chi}$, the mass of the $\chi$ field, and it is also much larger than the temperatures involved. We write then the couplings in terms of dimensionless ones via $g_{1}=\hat{g}_{1} m, g_{2}=\hat{g}_{2}$, and $g_{3}=\hat{g}_{3} m$. The Lagrangian (26) contains all the relevant and marginal operators: $g_{1} h \chi^{2}$ and $g_{3} h^{3}$ are relevant operators, while, for example, $g_{2} h^{2} \chi^{2}$ is marginal. We expect the relevant physics to be well described by this set of operators.

The field $\chi$ is subject to $Z_{2}$ symmetry. Having the Yang-Mills deconfining phase transition in mind, in the theory with static order parameter field we chose the symmetric phase to be at low temperatures, and thus the symmetry was broken for temperatures greater than a phase transition temperature. Accordingly, $m_{\chi}^{2}>0$ for $T<T_{c}$ and $m_{\chi}^{2}<0$ for $T>T_{c}$. As we anticipated though in the previous section, when the order parameter field is non-static we cannot simply choose the direction of symmetry restoration a priori. In four-dimensions a Bose-Einstein distribution emerges for $\chi$, and thus thermal fluctuations become important. Thermal fluctuations have the tendency to restore symmetry, destroying the possibility for the formation and existence of any physical condensate at high temperatures. The direction of the phase transition is therefore important, and is completely determined by the theory. In the following, we adopt the standard picture for the direction of the phase transition, consistent with the effect of higher order corrections to $m_{\chi}$ : symmetry restoration sets in at high temperatures [27]. This is one of the major differences between the theories with three- and four- dimensional order parameter field. 


\section{A. Diagramatics}

Based on Lagrangian (26) the diagrams contributing to the $h$ two-point function at one-loop order are shown on (77). Even though these are the same as in the three dimensional theory, here they are computed taking into account the time dependence of $\chi$. We can show, that finite temperature corrections from all diagrams that involve an $h$ loop are Boltzman suppressed due to the heaviness of $h$. Accordingly, the relevant diagrams in (17) for the singlet $h$ field are those on the first line: the first (tadpole A), the second (eye), and the third (tadpole B). Unlike in the three dimensional, time independent case, where UV divergent tadpoles have been removed by renormalization, in the four dimensional, time dependent case, these diagrams also provide non-negligible temperature contributions. Recall, that previously only the zero mode $h_{0}$ coupled in the $h \chi^{2}$-interaction. This is clearly not the situation here: all modes of $h$, and all Matsubara modes of $\chi$ participate in the interactions. The diagrams are evaluated using standard techniques of the imaginary time formalism [9].

$$
\underline{Q}=-\frac{g_{2}}{4} T \sum_{m} \int \frac{d^{3} k}{(2 \pi)^{3}} \frac{1}{\omega_{m}^{2}+\omega^{2}}=-\frac{g_{2}}{4} \int \frac{d^{3} k}{(2 \pi)^{3}} \frac{1}{2 \omega}(1+2 f(\omega / T)),
$$

where $\omega=\sqrt{\mathbf{k}^{2}+m_{\chi}{ }^{2}}$ is the energy, and $\omega_{m}=2 \pi m T$ is the Matsubara frequency of the internal $\chi$-line. The second line in (27) is the result of the frequency summation, and $f(x)=1 /\left(e^{x}-1\right)$ is the Bose-Einstein distribution function. The term proportional to the number 1 before the distribution function represents the vacuum contribution, and is divergent in the ultraviolet. This divergence is absorbed in the mass renormalization process. The second term contains no divergence, since it is regularized by the distribution function. We focus on the physics in the phase transition region, where $m_{\chi} \ll T$. Since the mass of the order parameter field, $m_{\chi}$, vanishes at $T_{c}$, we use the high temperature limit to obtain an analytic result for the $h$ self-energy contribution

$$
\Pi_{\text {tadpole }}^{A} \simeq \frac{g_{2}}{24} T^{2} .
$$

Similarly, the other tadpole contributes with

$$
\Pi_{\text {tadpole }}^{B} \simeq-\frac{g_{1} g_{3}}{24} \frac{T^{2}}{m^{2}} .
$$

These contributions are real and provide temperature dependence to the $h$ mass, not present in the three dimensional theory. In terms of dimensionless couplings

$$
\Pi_{\text {tadpole }}=\left(\hat{g}_{2}-\hat{g}_{1} \hat{g}_{3}\right) \frac{T^{2}}{24} .
$$

Contribution to the self-energy of $h$ from the eye diagram is

$$
\begin{aligned}
\bigcirc- & =\left(\frac{g_{1}}{2}\right)^{2} T \sum_{m} \int \frac{d^{3} k}{(2 \pi)^{3}} \frac{1}{\omega_{m}^{2}+\omega^{2}} \frac{1}{\left(\omega_{m}-\omega_{n}\right)^{2}+\omega^{2}} \\
& =\left(\frac{g_{1}}{2}\right)^{2} \int \frac{d^{3} k}{(2 \pi)^{3}} \frac{1}{\omega} \frac{1}{4 \omega^{2}+\omega_{n}^{2}}(1+2 f(\omega / T)) .
\end{aligned}
$$

Here $\omega_{n}$ is the Matsubara frequency of the external line and $\omega=\sqrt{\mathbf{k}^{2}+m_{\chi}{ }^{2}}$. We have restricted the study to zero external momentum. In order to obtain physical quantities from this real Euclidean integral we analytically continue this to Minkowski space. After the replacement $i \omega_{n} \rightarrow E+i \epsilon$ we obtain

$$
\Pi_{\text {eye }}(E)=-2\left(\frac{g_{1}}{2}\right)^{2} \int \frac{d^{3} k}{(2 \pi)^{3}} \frac{1}{\omega} \frac{1}{E^{2}-4 \omega^{2}}(1+2 f(\omega / T)) .
$$

Another significant difference with respect to the theory with time independent order parameter field is the existence of a real and an imaginary part of the self-energy, that contributes to the two-point function $\left[E^{2}-m^{2}-\Pi(E)\right]^{-1}$. Here $m$ is the tree level mass of $h$ and at one-loop order $\Pi=\Pi_{\text {tadpole }}+\Pi_{\text {eye }}$. The real and imaginary parts can be extracted from (32) by using

$$
\frac{1}{z \pm i \epsilon}=P \frac{1}{z} \mp i \pi \delta(z)
$$


Accordingly, the real part is given by the principal value of (32)

$$
\operatorname{Re} \Pi_{\text {eye }}(E)=-2\left(\frac{g_{1}}{2}\right)^{2} \frac{1}{2 \pi^{2}} P \int d k \frac{k^{2}}{\omega} \frac{1}{E^{2}-4 \omega^{2}}(1+2 f(\omega / T)) .
$$

The term proportional to the number 1 before the distribution function is again the vacuum contribution, divergent in the ultraviolet, and is removed by usual renormalization. This real part represents a shift in the mass squared of the $h$, and for $h$ at rest, through its definition, determines the pole mass $M$ :

$$
M^{2}-m^{2}-\Pi_{\text {tadpole }}-\operatorname{Re} \Pi_{\text {eye }}(E=M)=0 .
$$

Self-consistent numerical solutions of the above equation show that the large tree level mass is dominant, and loop corrections are negligibly small in the temperature range of interest, i.e. near the phase transition. Another interesting fact is, that $M \simeq m$ acts as an infrared cutoff guaranteeing the absence of infrared divergence for the pole mass. As a consequence, in this case there is no need for the resummation of higher order diagrams. An analytic expression in powers of $T^{2} / m^{2}$, and setting $m_{\chi}=0$, can thus be obtained in the region we work in, namely $m_{\chi} \ll T \ll m$,

$$
\operatorname{Re} \Pi_{\text {eye }}(E=m)=-2\left(\frac{g_{1}}{2}\right)^{2}\left(\frac{1}{6} \frac{T^{2}}{m^{2}}+\frac{4 \pi^{2}}{15} \frac{T^{4}}{m^{4}}\right) \simeq-\frac{\hat{g}_{1}^{2}}{12} m^{2} \frac{T^{2}}{m^{2}} .
$$

The absence of the infrared divergence in the pole mass is another relevant difference between the two theories discussed in this paper. This becomes apparent when summing all the one-loop contributions from (30) and (34):

$$
M^{2}=m^{2}\left[1+\left(\hat{g}_{2}-\hat{g}_{1} \hat{g}_{3}-2 \hat{g}_{1}^{2}\right) \frac{T^{2}}{24 m^{2}}+\mathcal{O}\left(\frac{T^{4}}{m^{4}}\right)\right] .
$$

The imaginary part of the self energy represents the net rate of $h$-decay, and its evaluation provides no difficulties:

$$
\operatorname{Im} \Pi_{\text {eye }}(E)=-2\left(\frac{g_{1}}{2}\right)^{2} \frac{1}{16 \pi} \Theta\left(E-2 m_{\chi}\right) \sqrt{1-\frac{4 m_{\chi}^{2}}{E^{2}}}(1+2 f(E / 2 T)) .
$$

By rewriting $(1+2 f)=(1+f)^{2}-f^{2}$ it is easy to understand that above the threshold, $E=2 m_{\chi}$, (38) determines the decay process $h \rightarrow \chi \chi$, and the annihilation $\chi \chi \rightarrow h$, weighted by the thermal distribution, with the net decay rate given by

$$
\Gamma=-\frac{\operatorname{Im} \Pi_{e y e}(E)}{2 E} .
$$

Unlike in the three dimensional case, here there are finite temperature corrections also to the two-point function of the now dynamic order parameter field. The full set of one-loop diagrams contributing to the $\chi$ field in the symmetric phase, together with their corresponding combinatorial factors is:

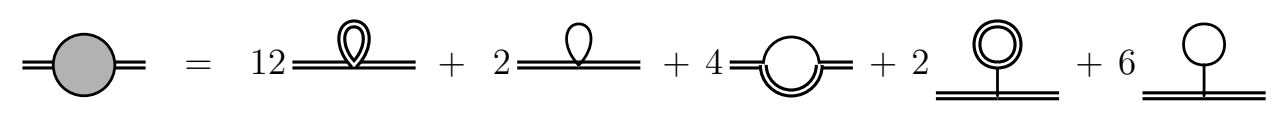

Due to the existence of a cubic $\chi$ self-coupling in the broken phase, there are, in addition to (40), two more diagrams:

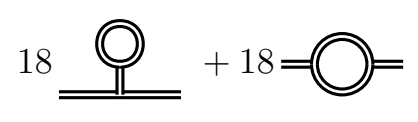

Here we are not going to discuss further details about the $\chi$ two-point function. The evaluation of these diagrams is standard. There are two points we wish to stress though: First, that contrary to the three dimensional theory, there are explicit thermal fluctuations providing temperature dependence to the mass of the order parameter field; Second, this mass is zero at the critical temperature of the second order phase transition, and has thus $m_{\chi}^{2} \propto\left(T_{c}-T\right)$ behavior. 


\section{B. Static Properties}

In the three-dimensional theory we found an infrared divergent result for the one-loop contribution to the $h$ twopoint function. This divergence came from what we call the eye diagram. In four dimensions, as we have shown above, besides the eye diagram there are two tadpoles also contributing. Here we show that the static limit of the two-point $h$ function for the time dependent order parameter field displays the same features as for the time independent order parameter field case.

Here it is important to distinguish between the pole mass and the screening mass. The pole mass, $M$, is defined through (35) as the pole in the full two-point function. The screening mass, $m_{s}$, is defined by the location of the pole in the static propagator for complex momentum $p=i m_{s}$,

$$
p^{2}+\Pi(E=0, p)=0 .
$$

In the small momentum limit this leads to the following definition

$$
m_{s}^{2}=m^{2}+\lim _{p \rightarrow 0} \Pi(E=0, p) .
$$

In three dimensions the pole and screening masses are one and the same thing, since the static propagator is the full propagator.

We have shown, that when looking at the pole mass the IR problem of the eye diagram is regulated by the heavy $h$ mass. When analyzing the screening mass, however, the one-loop IR divergence present in the three dimensional case is recovered. Based on the above definition, we set $E=0$ in expression (34). This corresponds to effectively reducing the 4 dimensional theory to a 3 dimensional one. To display the relevant infrared contribution we take the high temperature expansion in (34) which yields:

$$
2\left(\frac{g_{1}}{2}\right)^{2} \frac{T}{4 \pi^{2}} \int_{0}^{\infty} d p \frac{p^{2}}{\left(p^{2}+m_{\chi}^{2}\right)^{2}}=g_{1}^{2} \frac{T}{32 \pi m_{\chi}} .
$$

Hence, for the static limit the phase transition region is dominated by the same type of infrared divergence we encountered in the time independent order parameter field case. Note, however, that the numerical constant in eq. (44) differs from that in the second term of eq. (9). The reason for this is that the reduction, both here and in section III was done only for the modes of $h$, and thus in eq. (44), contrary to (9), all Matsubara modes of $\chi$ contribute.

By combining all the diagrams we find for the screening mass at one-loop order

$$
m^{2}\left[1-\frac{\hat{g}_{1}^{2}}{32 \pi} \frac{T}{m_{\chi}}-\left(\hat{g}_{1} \hat{g}_{3}-\hat{g}_{2}\right) \frac{T^{2}}{24 m^{2}}\right],
$$

showing clearly the eye contribution as the infrared dominant one. Note, that above we have tree level coefficients. A complete investigation would require renormalization group analysis, but this is beyond the scope of this paper. We thus recovered our universal behavior from before.

\section{CONCLUSIONS}

When analyzing many physical situations, it is common practice to isolate the order parameter field, and more generally, the light degrees of freedom, since these are expected to be the relevant states at low energies. While this procedure certainly is reasonable, in nature most of the physical fields are neither order parameter fields, nor light at all. In order to extract information from these heavy states, we needed first to determine new and universal features associated with them. We have used a general strategy proposed first in 1, 2], according to which we couple the light degrees/order parameter fields to the heavy fields in the most general way, and then truncate the theory by retaining all the relevant and marginal operators in the Lagrangian. In doing so, the theory is fully renormalizable while capturing the relevant contributions. The operator set is further constrained by imposing all the relevant symmetries of the problem at hand. In order for our procedure to work, we also assume, that the other physical states of the theory have masses larger than our non-order parameter field. In this way we can, formally, integrate these states out, and their effects are absorbed in the modified couplings of our effective Lagrangian.

For both the time independent and time dependent order parameter field we have shown, that the spatial correlators of the non-order parameter field are infrared dominated, and hence can be used to determine the properties of the phase transition. We have determined the general behavior of the screening mass of a generic singlet field, and have shown how to extract all the relevant information from such a quantity. Somewhat surprisingly, we have 
demonstrated, that the pole mass of any non-order parameter physical field is not infrared dominated. Our results can be immediately applied to any generic phase transition. We have used as relevant example, for the time independent order parameter field case, the deconfining transition of Yang-Mills theories as also explained in [1, 2]. Lattice simulations [6] for the deconfining phase transition support our results. Different approaches have been used in literature to study the deconfining phase transition 1, 10, 11, 12, 13, 14, 15, 16, 17, 18, 19, 20, 21, 22. Here we have been able to unify some of them. In fact, by demonstrating that there exists an extended universal behavior for singlet fields, we have uncovered the relation between phenomenologically oriented models which use the glueball Lagrangian 11, 12, 13, 15, 21 to describe the deconfining phase transition, and the ones using the symmetries of the Polyakov loops [16, 17, 18, 23, 24, 25].

The induced critical behavior is universal, however the quantitative details depend on the strength of the couplings between the fields as well as the resummation procedure. We have considered only one non-order parameter field, but many are expected to display a similar behavior. For the Yang-Mills deconfining phase transition lattice QCD simulations are able to determine the coupling strength of any glueball state to the Polyakov loop by following the temperature dependence of screening masses of such states.

Our analysis suggests, that monitoring a number of spatial correlators, or more specifically their derivatives, is an efficient and sufficient way to experimentally uncover the chiral/deconfining phase transition and its features.

\section{Acknowledgments}

It is a pleasure to thank P.H. Damgaard, A.D. Jackson, J. Kapusta, C. Marchetti, and J. Schechter for discussions and careful reading of the manuscript. We acknowledge R. Pisarski for insightful discussions. Comments by E. Kolomeitsev are appreciated. The work of F.S. is supported by the Marie-Curie fellowship under contract MCFI2001-00181.

[1] F. Sannino, Phys. Rev. D 66, 034013 (2002) arXiv:hep-ph/0204174.

[2] A. Mocsy, F. Sannino and K. Tuominen, arXiv:hep-ph/0301229 to appear in PRL.

[3] P.H. Damgaard, Phys. Lett. B194 (1987) 107; J. Kiskis, Phys. Rev. D41 (1990) 3204; J. Fingberg et al., Phys. Lett. B248 (1990) 347; J. Christensen and P.H. Damgaard, Nucl. Phys. B348 (1991) 226; P.H. Damgaard and M. Hasenbush, Phys. Lett. B331 (1994) 400; J. Kiskis and P. Vranas, Phys. Rev. D49 (1994) 528.

[4] S. Hands, Nucl. Phys. Proc. Suppl. 106, 142 (2002) arXiv:hep-lat/0109034.

[5] S. R. Coleman, R. Jackiw and H. D. Politzer, Phys. Rev. D 10, 2491 (1974).

[6] For early studies on the relation between glueballs and Polyakov loops see: P. Bacilieri et al. [Ape Collaboration], Phys. Lett. B 220, 607 (1989).

[7] S. Weinberg, Phys. Rev. D9, 3357 (1974).

[8] R.N. Mohapatra and G. Senjanović, Phys. Rev. Lett. 42, 1651 (1979); Phys. Rev. D20, 3390 (1979).

[9] J. I. Kapusta, Finite-temperature Field Theory, Cambridge University Press, Cambridge (1989).

[10] G. Boyd et al., Nucl. Phys. B 469, 419 (1996) arXiv:hep-lat/9602007.

[11] N. O. Agasian, JETP Lett. 57, 208 (1993) [Pisma Zh. Eksp. Teor. Fiz. 57, 200 (1993)].

[12] B. A. Campbell, J. R. Ellis and K. A. Olive, Nucl. Phys. B 345, 57 (1990).

[13] Yu. A. Simonov, JETP Lett. 55, 627 (1992) [Pisma Zh. Eksp. Teor. Fiz. 55, 605 (1992)].

[14] J. Sollfrank and U. W. Heinz, Z. Phys. C 65, 111 (1995) arXiv:nucl-th/9406014.

[15] G. W. Carter, O. Scavenius, I. N. Mishustin and P. J. Ellis, Phys. Rev. C 61, 045206 (2000) arXiv:nucl-th/9812014.

[16] R. D. Pisarski, Nucl. Phys. A 702, 151 (2002) arXiv:hep-ph/0112037; Phys. Rev. D 62, 111501 (2000) arXiv:hep-ph/0006205.

[17] A. Dumitru and R. D. Pisarski, Phys. Lett. B 525, 95 (2002) arXiv:hep-ph/0106176; Phys. Lett. B 504, 282 (2001) arXiv:hep-ph/0010083; P. N. Meisinger, T. R. Miller and M. C. Ogilvie, Phys. Rev. D 65, 034009 (2002) arXiv:hep-ph/0108009 ; P. N. Meisinger and M. C. Ogilvie, Phys. Rev. D 65, 056013 (2002) arXiv:hep-ph/0108026.

[18] C. P. Korthals Altes, R. D. Pisarski and A. Sinkovics, Phys. Rev. D 61, 056007 (2000) arXiv:hep-ph/9904305.

[19] J. Wirstam, Phys. Rev. D 65, 014020 (2002) arXiv:hep-ph/0106141.

[20] M. Laine and O. Philipsen, Phys. Lett. B 459, 259 (1999) arXiv:hep-lat/9905004.

[21] F. Sannino, N. Marchal and W. Schafer, Phys. Rev. D 66, 016007 (2002) arXiv:hep-ph/0202248.

[22] N. O. Agasian, Phys. Lett. B 562, 257 (2003) arXiv:hep-ph/0303127.

[23] B. Svetitsky and L. G. Yaffe, Nucl. Phys. B 210, 423 (1982); L. G. Yaffe and B. Svetitsky, Phys. Rev. D 26, 963 (1982); B. Svetitsky, Phys. Rept. 132, 1 (1986).

[24] O. Scavenius, A. Dumitru and A. D. Jackson, Phys. Rev. Lett. 87, 182302 (2001) arXiv:hep-ph/0103219.

[25] O. Scavenius, A. Dumitru and J. T. Lenaghan, Phys. Rev. C 66, 034903 (2002) arXiv:hep-ph/0201079.

[26] P. N. Meisinger and M. C. Ogilvie, Phys. Rev. D 66, 105006 (2002) arXiv:hep-ph/0206181. 
[27] There are however exceptions as discussed in [7, 8] 


$$
\begin{aligned}
- & -= \\
& =12 \frac{0}{}+2-\left(18-O^{-}\right.
\end{aligned}
$$

